\title{
Challenges of Sustaining the International Space Station through 2020 and Beyond: Including Epistemic Uncertainty in Reassessing Confidence Targets
}

\author{
Leif Anderson ${ }^{1}$ \\ Manufacturing Technical Solutions, Inc., Houston, Texas, 77059 \\ Katrina Carter-Journet ${ }^{2}$ and Neil Box ${ }^{3}$ \\ ARES Corporation, Houston, Texas, 77058 \\ Denise DiFilippo ${ }^{4}$ and Sean Harrington ${ }^{5}$ \\ The Boeing Company, Houston, Texas, 77059 \\ David Jackson ${ }^{6}$ and Michael Lutomski ${ }^{7}$ \\ National Aeronautics and Space Administration (Johnson Space Center), Houston, Texas, 77058
}

\begin{abstract}
This paper introduces an analytical approach, Probability and Confidence Trade-space (PACT), which can be used to assess uncertainty in International Space Station (ISS) hardware sparing necessary to extend the life of the vehicle. There are several key areas under consideration in this research. We investigate what sparing confidence targets may be reasonable to ensure vehicle survivability and for completion of science on the ISS. The results of the analysis will provide a methodological basis for reassessing vehicle subsystem confidence targets. An ongoing annual analysis currently compares the probability of existing spares exceeding the total expected unit demand of the Orbital Replacement Unit (ORU) in functional hierarchies approximating the vehicle subsystems. In cases where the functional hierarchies' availability does not meet subsystem confidence targets, the current sparing analysis further identifies which ORUs may require additional spares to extend the life of the ISS. The resulting probability is dependent upon hardware reliability estimates. However, the ISS hardware fleet carries considerable epistemic uncertainty (uncertainty in the knowledge of the true hardware failure rate), which does not currently factor into the annual sparing analysis. The existing confidence targets may be conservative. This paper will also discuss how confidence targets may be relaxed based on the inclusion of epistemic uncertainty for each ORU. The paper will conclude with strengths and limitations for implementing the analytical approach in sustaining the ISS through end of life, 2020 and beyond.
\end{abstract}

\footnotetext{
${ }^{1}$ Technical Engineer, ISS Supportability Analysis \& Supply Support, MTS Inc, 13100 Space Center Blvd, MC HB3-30, Houston, Texas, 77059, and AIAA Member.

${ }^{2}$ Senior Engineer/Scientist, 2101 NASA Parkway, Houston, Texas 77058/JSC:OE (4S/3906F), and AIAA Member.

${ }^{3}$ Probabilistic Risk Analyst/Electrical Engineer, 2101 NASA Parkway, Houston, Texas 77058/JSC:OE (4S/3909D).

4 Technical Lead Engineer, ISS Supportability Analysis \& Supply Support, The Boeing Company, 13100 Space Center Blvd, MC HB3-30, Houston, TX, 77059.

${ }^{5}$ Technical Engineer, ISS Supportability Analysis \& Supply Support, The Boeing Company, 13100 Space Center Blvd, MC HB3-30, Houston, TX, 77059, and AIAA Member.

6 ISS Strategic and Tactical Logistics and Maintenance Lead, 2101 NASA Parkway, Houston, Texas 77058/JSC:OB5.

7 Risk Manager, International Space Station Safety \& Mission Assurance/Risk Office, 2101 NASA Parkway, Houston, Texas, 77058/JSC:OE.
} 


\section{Introduction}

The International Space Station (ISS), as shown in Figure 1, was originally designed to operate until 2015. Currently, the international partnership has agreed to extend operations until 2020 and have further agreed not to take any actions that would prevent extending the mission beyond 2020. Each international partner is responsible for the sustaining engineering, sparing, and maintenance of their own segments. The National Aeronautics and Space Administration's (NASA's) challenge is to provide sufficient resources to maintain the functional availability of the ISS systems in the United States On-Orbit Segments (USOS).

Prior to retirement of the Space Shuttle, spares had already been purchased and in many cases prepositioned on-orbit to meet the critical ISS maintenance requirements through the previously expected end of life of 2015. However, with the

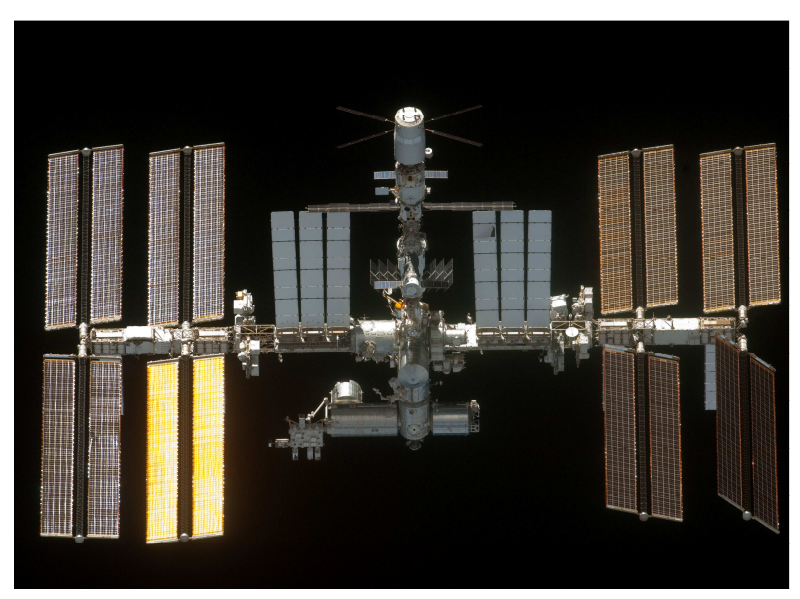

Figure 1. Overall View of ISS. Figure shows overall view of the International Space Station (ISS) taken as the shuttle Endeavour departed at the end of the STS-134 mission (6).

ISS operating to an uncertain date in the future, the Program will now be in a continuous sustaining effort to extend the life of the ISS. This sustaining effort must succeed in a highly resource constrained environment (supply vehicles, crew-time, spares quantities, and budget are all potentially constraining). While long lead times may suggest the advisability of early decision-making (to procure additional maintenance spares), experience suggests that such decisions should be delayed as long as it is reasonable to do so. Delaying procurement decisions allows the program to gather additional performance data on the ISS components and spares and thus make better decisions. When assessing maintenance demands, the Program considers that some graceful degradation of functional redundancy may be acceptable near end of vehicle life.

The methodology for assessing spares requirements for maintenance is continuously reviewed and improved. One area that has not previously been quantified in maintenance analysis is the epistemic uncertainty inherent in the reliability estimates for the ISS hardware. While this risk has been implicitly understood, its impact had not been explicitly included in the maintenance assessments.

This paper introduces application of an analytical approach to assessing this epistemic uncertainty in component or sparing reliability rates of ISS hardware necessary to extend the life of the vehicle. For a given component or group of components or spares, we look at trading a target probability of sufficient spares with the confidence in that target probability. This paper will conclude with strengths, limitations, and future challenges for implementing the analytical approach, Probability and Confidence Trade-space (PACT), for use in sustaining the ISS vehicle.

\section{Key Concepts and Definitions}

The following sub-sections will briefly define uncertainty and other key definitions that will be used in other sections of the manuscript.

\section{A. Sources of Uncertainty}

Uncertainty can be categorized as two distinct types: aleatoric and epistemic.

Aleatoric (or stochastic) uncertainty is indicative of natural, intrinsic randomness (variability) in a physical process. Aleatoric uncertainty cannot be suppressed by more accurate measurements and is irreducible uncertainty (1).

Epistemic uncertainty (or reducible uncertainty) is due to a lack of knowledge (or data) about the processes, models, parameters (e.g. failure rate), and behavior used in the analysis. "Failure rates are uncertain, sometimes 
because failure information is sparse or unavailable, and sometimes because the very applicability of available data to the case at hand may be in doubt (1)." Only epistemic uncertainty is reducible through operational experience.

It is important that both natural variability of physical processes (i.e., aleatory or stochastic uncertainty) and the uncertainties in knowledge of these processes (i.e., "epistemic" or state-of-knowledge uncertainty) are properly accounted for (1). In practical applications, both kinds of uncertainties are almost always present.

\section{B. Model Parameters}

Confidence is a measure of the fidelity of an estimate. Confidence indicates the soundness of the estimate. However, confidence does not predict the true value of a parameter.

Error Factor (EF) is the ratio of the upper 95th percentile to 50th percentile (median) in a lognormal distribution. Greater values of the EF correspond to a wider distribution and greater uncertainty. A narrow distribution, as EF approaches one, implies more confidence.

Operational Failure Rate is the expected number of inherent failures of a part (or ORU) during operation per unit time. The operational failure rate is determined by Bayesian analysis using original reliability predictions, observed operating time and failure history, and assumed Error Factor.

Probability of Sufficiency (POS) is the likelihood that sufficient spares exist to repair all failures. "Whenever possible, the specific spare parts and quantities proposed should be based upon probabilistic equations using parameters such as predicted/designed consumption rates, available empirical data, engineering estimates of predicted use of the spare parts, and the overall probability of sufficiency (POS) assumed ... POS is the probability that the spare parts will be available when needed (2)."

Confidence Target is the desired confidence in POS value. Confidence assesses the uncertainty around the POS. For instance, instead of saying that the POS is $90 \%$ for a given Orbital Replacement Unit (ORU), one can say for example that there is a $50 \%$ chance that the POS is at least $90 \%$. This added dimension gives insight into the uncertainty of calculations. Confidence is expressed as a percentage where $100 \%$ implies total certainty or full confidence.

\section{Uncertainty and Risk in ISS Maintenance Projections}

How many equipment failures will the ISS experience this year? How many over its lifetime? Which equipment will fail? When will it fail? What is the lifetime of the ISS?

These are the questions that must be answered in order to safely and successfully sustain the orbiting complex of laboratories, environmental systems and utility systems that comprise the ISS.

Fundamentally, every project, plant, business, etc., faces the same sorts of questions. ISS faces some unique challenges, among them: Unique hardware, small populations, challenging delivery channels, harsh environments, limited storage space, and limited crew time to perform repairs.

ISS Logistics and Maintenance (L\&M) has taken a sparing approach that aggressively protects for success through end of life (currently assumed to be 2020, though analysis through 2028 has been performed) in an annual Sparing Analysis. ORU failure rates are based on both original predictions using MIL-STD 217 (3) and operational experience (via Bayesian analysis). Monte Carlo simulations distribute failures around a range of values assuming an exponential distribution and are used to project failure impacts across vehicle life. A large number of simulations are performed (typically 1000); confidence that the vehicle systems meet at least minimum required functional targets is determined by counting the percentage of the total simulations that meet or exceed the target minimum configuration. Spares are deemed sufficient when the confidence percentage meets or exceeds a specified confidence target. Historically, these targets have been conservative, ranging between $85 \%$ and $99.5 \%$. 
This approach represents the natural variability present in hardware reliability; that is the inherent, or aleatory, uncertainty that is present when evaluating failure projections and spares sufficiency. This inherent uncertainty persists regardless of increases in knowledge; it is not reducible.

This approach includes an implied assumption that the failure rate estimate used in simulation is the true failure rate of the equipment. In reality, there is also uncertainty as to the true value of the failure rate. This represents a primary source of epistemic uncertainty (uncertainty due to incomplete knowledge). Epistemic uncertainty is reducible with increased knowledge. For ISS ORU failure rates, this increased knowledge derives from operating experience (as opposed to life testing that might be performed for equipment that is less expensive or more widely used).

Due to the small populations, as well as uniqueness of the hardware and operating environment, there is significant uncertainty in determining true failure rate. Current methods bound the failure rate estimates, but the bounds are not taken into account in the simulations and calculations. Upper and lower bounds are characterized by an error factor. Initial error factors were assumed program wide (to be equal to 4). The application of Bayesian analysis to the predicted failure rate and observed operating experience (operating hours and failure history) results in an improved estimate of failure rate and reduces epistemic uncertainty to the extent that operating hours and failure history increase knowledge.

Outputs of the Bayesian update process also include a posterior error factor. A decreasing posterior error factor quantifies the extent to which epistemic uncertainty has been reduced. The error factor also impacts the size and shape of the trade space of sufficiency and confidence (this will be illustrated in a later section). Note that some uncertainty remains for any error factor other than one (where error factor equal to one represents complete certainty of the true failure rate, and is not realistically attainable).

There may also be other sources of epistemic uncertainty in the failure distribution. For example, the actual distribution may not be exponential. Also, as yet undiscovered wear-out modes may exist that might influence the failure distribution.

The calculated confidence numbers of the current methodology can thus be shown to be incomplete since they do not take into account epistemic uncertainty. In the past, this has driven high targets to compensate. If confidence is determined by taking into account both aleatory and epistemic uncertainty, then less extreme targets may be acceptable and still support successful maintenance of the ISS through end of program life.

\section{Overview of Current Approach to Sparing Assessments}

The current practice for the ISS sparing assessments is a combination of quantitative analysis and engineering judgment annual assessment involves intense coordination among engineering teams and program boards. The Logistics \& Maintenance (L\&M) office in the Vehicle directorate at Johnson Space Center sponsors this activity. The findings from a sparing assessment have wide application, from supplementing periodic Original Equipment Manufacturer decision packages, to supporting Government Accountability Office audit (4). The assessment gives indication of the risk in supporting the vehicle life.

As of 2011, the L\&M annual sparing assessment models approximately 30 vehicle sub-systems (for example thermal control, guidance and navigation, environmental control and life support), which comprises approximately 300 unique ORUs. Roughly half of the sub-systems are modeled as groups of ORUs with hierarchal operation through vehicle life in a discrete event simulation tool. These functions are subject to a confidence target. Where the ORU does not meet a level availability to provide for the function-wide target at minimum configuration, additional ORU spares are identified. Alternatively, the remaining sub-system ORUs are modeled as stand-alone hardware where the failure of one ORU does not impact the operation of any other hardware. These ORUs are subject to target thresholds for determining additional spares as well, but with a simpler approach to measure ORU availability. This sub-set of ORUs will be used to describe the process of identifying appropriate sparing for the entire population as the process can be applied to the entire population. Figure 2 illustrates the current process flow. 


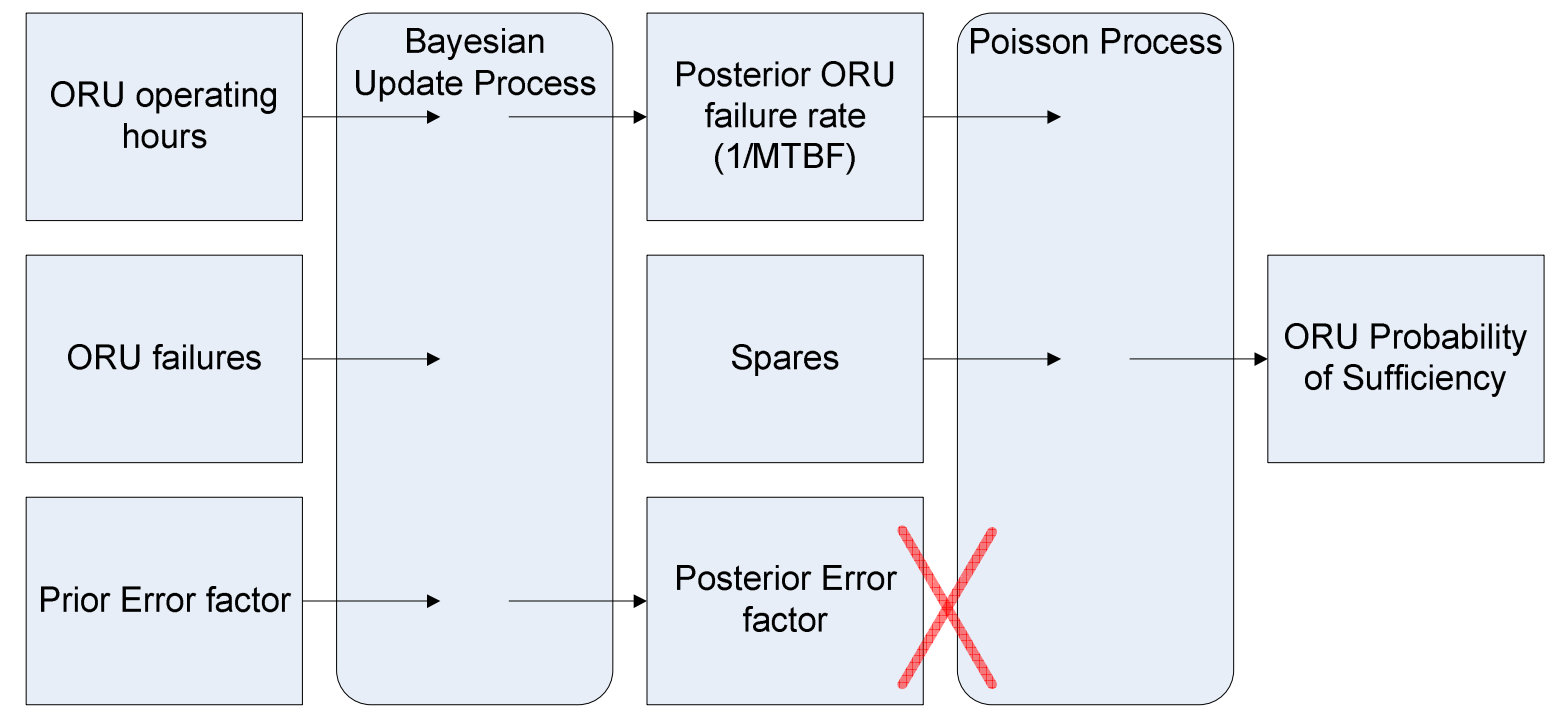

Figure 2. Current Process Flow. Figure shows flowchart for the current process flow used to obtain the ORU Probability of Sufficiency (POS). The posterior error factor is not input in the Poisson Process.

Underlying the sparing assessment is a Bayesian update process on the ORU failure rates. The ISS specific application of Bayesian inference updates the predicted ORU failure rate (prior) given operational experience resulting in an operational (posterior) ORU failure rate. Operational experience is defined as the time (e.g. number of hours) an ORU has been activated and the number of random failures observed. A prior and posterior error factor (EF) are parameters to the Bayesian update process. The EF weighs ORU operational experience against the predicted ORU failure rate. The ISS Program currently uses a prior EF equal to four. The process flow chart (Figure 2) indicates that the operational ORU failure rate is then used as input into a Poisson process. This is a statistical technique based on the cumulative Poisson probability distribution estimating the probability that ORU failures in a projected time period do not exceed the number of ORU spares. Hence, we are provided an indication of the ORU probability of sufficiency (POS). This estimate is compared against an arbitrary target probability that varies from ORU to ORU, function to function. Conceptually, the POS estimate can be represented as a single dimensional value, ranged $0-100 \%$, at a given point in time, compared to a desired target, as shown in Figure 3. The Poisson process is not equipped to define the confidence in an ORU POS value. Instead, we infer absolute confidence in the estimate. 


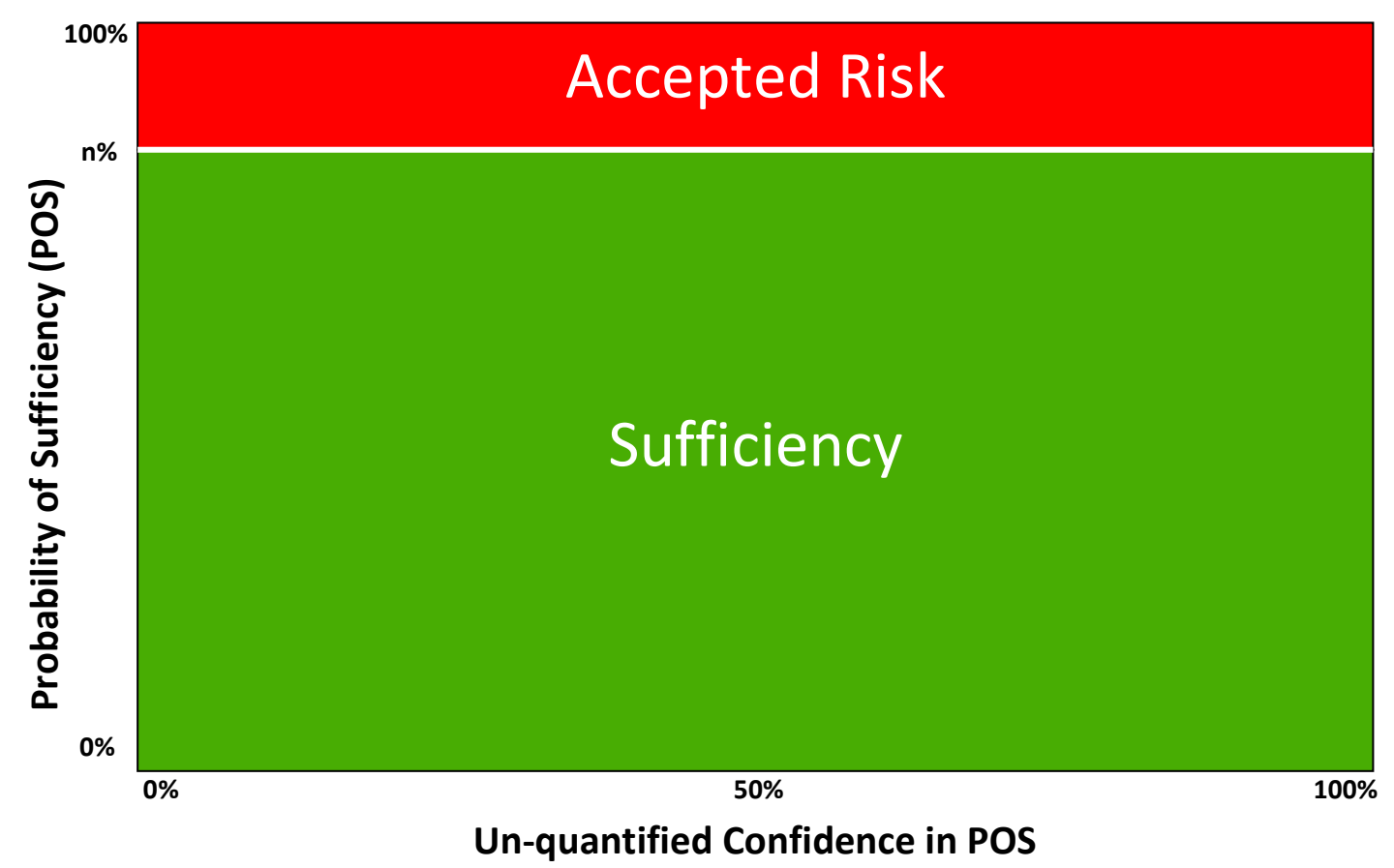

Figure 3. Current Model of ISS Sparing Risk. Figure shows graphical interpretation of the current sparing approach.

This process has proven useful, for it distinguishes an area of 'Accepted Risk' in the ORU sufficiency at a given point in time, and an area of ORU sufficiency, with probability value $\mathrm{n} \%$ as shown in Figure 3. This provides insight for risk informed decision making.

Figure 3 includes a straight line cutting through the probability/confidence region. Confidence along this line can be interpreted as absolute; POS is independent of confidence. This is a limitation in the current practice. With modification of the process, the region can be used to display the epistemic uncertainty in ORU failure rates. Intrinsic to every POS estimate there is epistemic uncertainty in the ORU failure rate that the Poisson process does not capture. This uncertainty can be represented as a confidence in the probability estimate. The confidence allows a second dimension to be quantified as shown in Figure 5 of the following section.

We demonstrate this approach with a generic standalone ORU; its failure rate 1.00E-5, a projected time period of 9 years, and its baselined number of spares, 1. This example can be replicated in Excel or using most statistical software.

Given these input parameter values, the ORU POS is $81 \%$ through 9 years. If we were to consider meeting a target desired by the program in that time frame of $95 \%$, one additional spare would be indicated in the assessment. Importantly, this number is typically not used as a procurement recommendation. Other factors are considered such as budget, procurement lead-times, manifest constraints, and repair capability. The ISS Spares Assessment is a coordinated effort involving many stakeholders such as engineering experts, suppliers, and program management. Since we have uncertainty in the ORU failure rate, a fundamental input to the model, various considerations accommodate risk mitigation postures that could initiate operational strategies, redesign work, or prioritization on return flights to support ORU, function and ultimately, vehicle configuration through end of life. 


\section{Overview and Discussion of the Proposed Approach}

By including epistemic uncertainty, we construct a more complete model.

Figure 4 illustrates modifications to current practice which allows us to do so. This approach is called Probability and Confidence Trade-space (PACT) Evaluation.

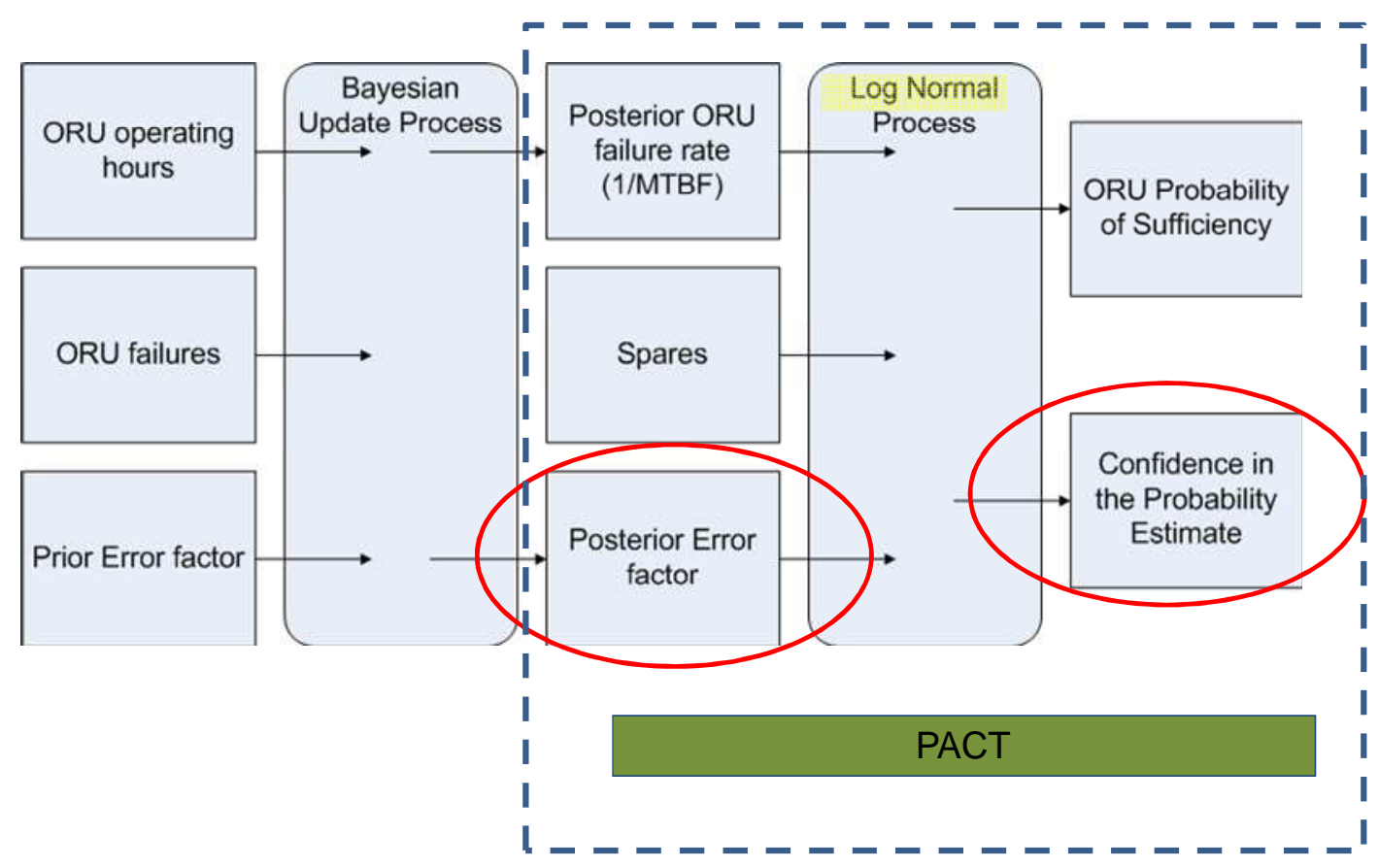

Figure 4. PACT: Proposed Process Flow. Figure shows flowchart for the current process flow used to obtain the ORU Probability of Sufficiency (POS) and confidence in the probability estimate.

In PACT work flow, the Bayesian update process remains unchanged. However, the posterior EF is now integrated into a Lognormal process. The Lognormal process is a statistical technique similar to the Poisson process but includes an additional parameter (EF) for determining the distribution variance in ORU failure rate.

Higher EF values reflect higher levels of uncertainty in the ORU failure rate; whereas lower EF values will narrow the distribution variance, thus reflect greater certainty. This added parameter allows for variation in the level of confidence in the ORU POS and thereby accounts for the epistemic uncertainty in the ORU failure rate.

\section{PACT Trade Space Regions}

The regions that describe current sparing risk; accepted risk and sufficiency, are no longer linear. The probability of sufficiency is no longer a straight line; it is now a curve. Furthermore, the space includes more nuanced regions: 1) The area of Sufficiency, 2) The area of Previously Accepted Risk, 3) the Area of Previously Accepted Risk that is now 'sufficient', and 4) The area of previously unidentified epistemic uncertainty. Figure 5 compares PACT Evaluation to the current approach (see Figure 3) in the Probability/Confidence trade-space. 


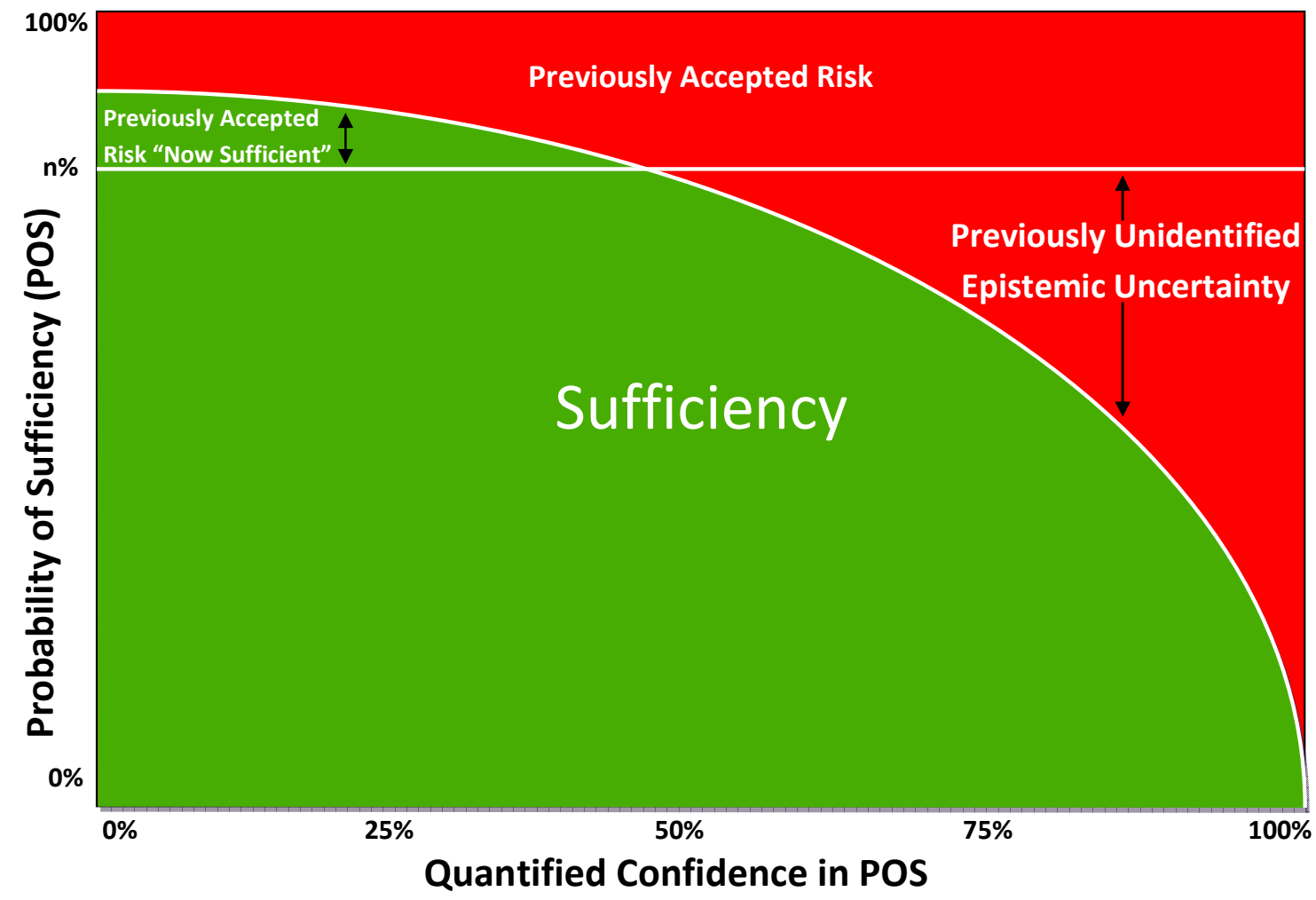

Figure 5. PACT Model of ISS Sparing Risk. Figure shows graphical interpretation of the PACT sparing approach and trade space.

Using PACT Evaluation results in a range of POS values with corresponding confidence values. Figure 6 shows the PACT Evaluation results using the generic ORU example presented in Section IV. The ORU POS values are plotted on the y-axis and corresponding confidence levels are plotted on the x-axis. For example, recall that under the Poisson process approach we estimate the ORU POS to be $81 \%$ through 9 years. In Figure 6 we see that when accounting for epistemic uncertainty using PACT evaluation we are approximately $70 \%$ confident in that estimate. 


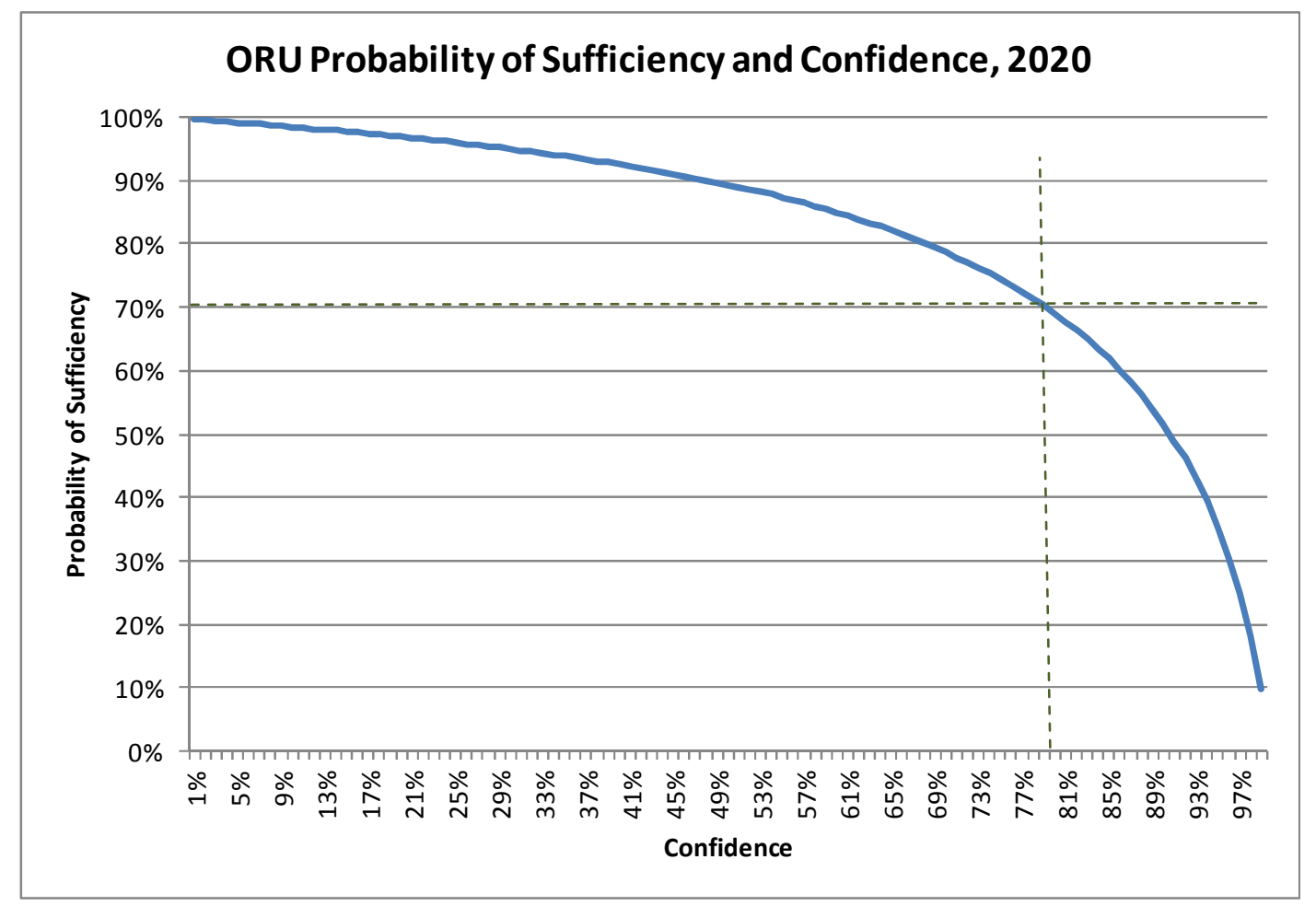

Figure 6 Generic ORU Example. Figure shows graphical application of the PACT evaluation on a generic ORU.

\section{Interpretation of Trade Space Regions}

With this knowledge, we can assess the tradeoff along the curve between the desired ORU POS value and our confidence in that value. For a sparing assessment this tradeoff can be summarized with two options based on quantitative assessment:

1) We may accept the probability and our confidence as is,

2) We may relax our confidence for a higher desirable POS value, or we may consider a lower desirable POS value in favor of having higher confidence.

Alternately, we may influence the ORU POS and confidence estimates by assuming additional spares (through procurement or repair of failed units) or by changing the projected horizon time for the analysis.

The last option has a unique implication. The current spares assessment approach has fixed thresholds throughout vehicle life. Since we can now control confidence levels through PACT Evaluation, we are in a position to acknowledge lower certainty with time with our knowledge of the ORU 'true' failure rate and thereby accept lower confidence levels at later stages of a projection. 
Figure 7 illustrates the point using the generic ORU as example. This graph shifts the $\mathrm{x}$-axis to time and displays the resulting confidence in a fixed POS value, 90\%. It is evident that our confidence in the POS estimate is higher in the near-term versus projected end of life scenarios. This dynamic becomes a key issue in discussions for adopting PACT Evaluation in future sparing assessments in the ISS program, topic of Section VI.

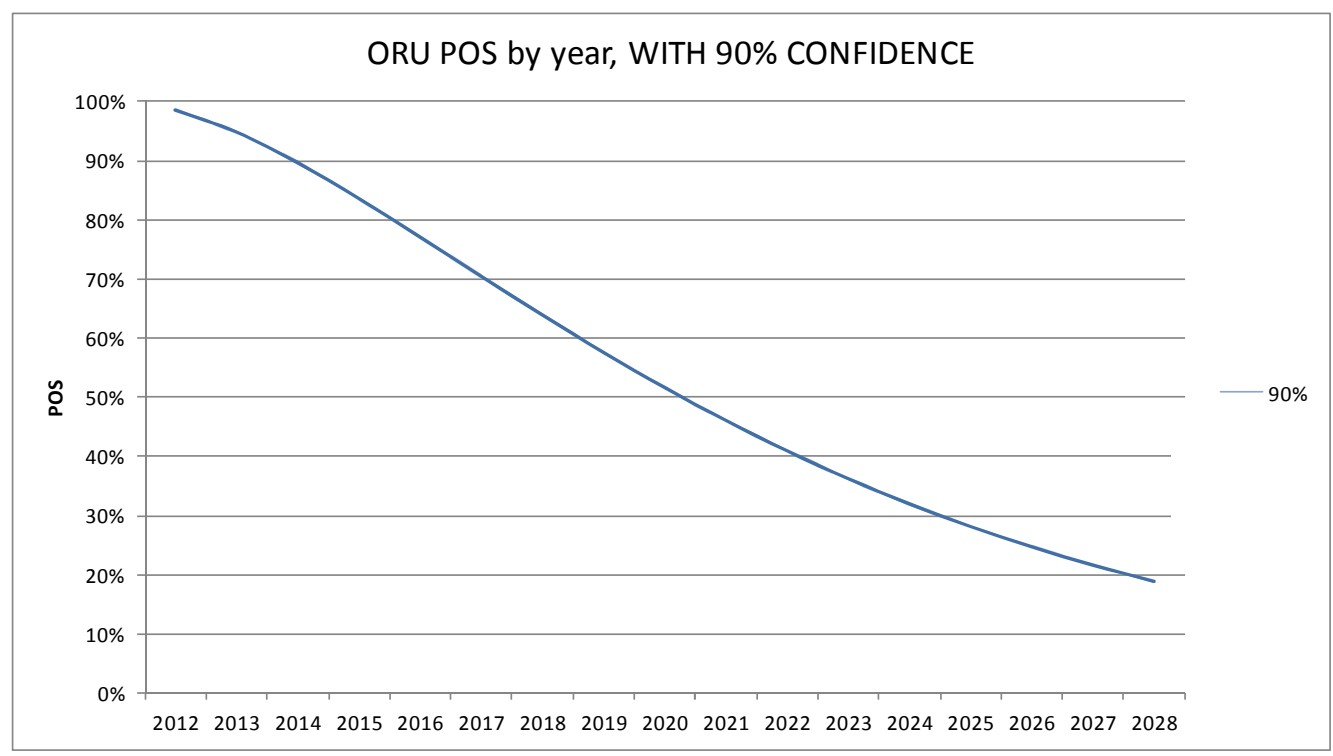

Figure 7. Generic ORU time-series example. Figure shows ORU POS 2012-2028 if we were want to maintain $90 \%$ confidence in that estimate.

\section{E. Case Study Results}

Six ORUs, representing a variety of ISS supportability challenges/risk imparting sparing levels, were selected for a case study to validate the usefulness of PACT. The six ORUs are shown in Appendix A. Analysis of the six ORUs demonstrates a breadth of uncertainty; empirical basis of ORU failure rate demonstrated versus lack of operational experience. The results for the ORU case studies can be seen in Appendix A.

\section{Application of PACT Evaluation for Revising ISS Sparing Confidence Targets}

The current approach to ISS sparing analysis protects for the epistemic uncertainty in the ORU failure rate by adopting high confidence targets. These targets range from $85 \%$ to above $99 \%$. The primary disadvantage of this approach, when incorporated with a Poisson process, is resulting sparing demand inflation over time. To illustrate this characterization we can project out in time using the hypothetical ORU presented in Section IV and identify the necessary number of spares needed to reach a 95\% POS target. For a projection beginning in 2012, with the one baseline spare, at this target level, one additional spare is needed through 2016. Two additional spares are needed through 2021. This number increases to 3 additional spares through 2028. Whereas, the expected number of failures through 2016, 2021, and 2028 is $0.44,0.88$, and 1.49 failures, respectively. 
When the ORU target POS remains constant, the trade-off is clear; we identify needing a number of spares that may exceed any reasonable expectation of the number of failures that will actually be observed. This effect becomes more pronounced as the time horizon increases. When supplementing program decision-making for hardware investment, this may reduce the usefulness of sparing assessment results. Program management must rely on expert engineering judgment. This situation ultimately cannot nor should it be entirely eliminated. However, using an approach similar to PACT Evaluation, we are able to vary our confidence levels and thus improve the utility of the sparing assessment results. It may be appropriate and useful to coordinate with Program Management and the System teams to accept for instance near, mid, and long -term targets for both ORU POS and the confidence level. Figure 8 illustrates the following example levels: $90 \%$ POS and confidence in the near-term, $75 \%$ POS and confidence in the mid-term, and $60 \%$ POS and confidence in the long-term.

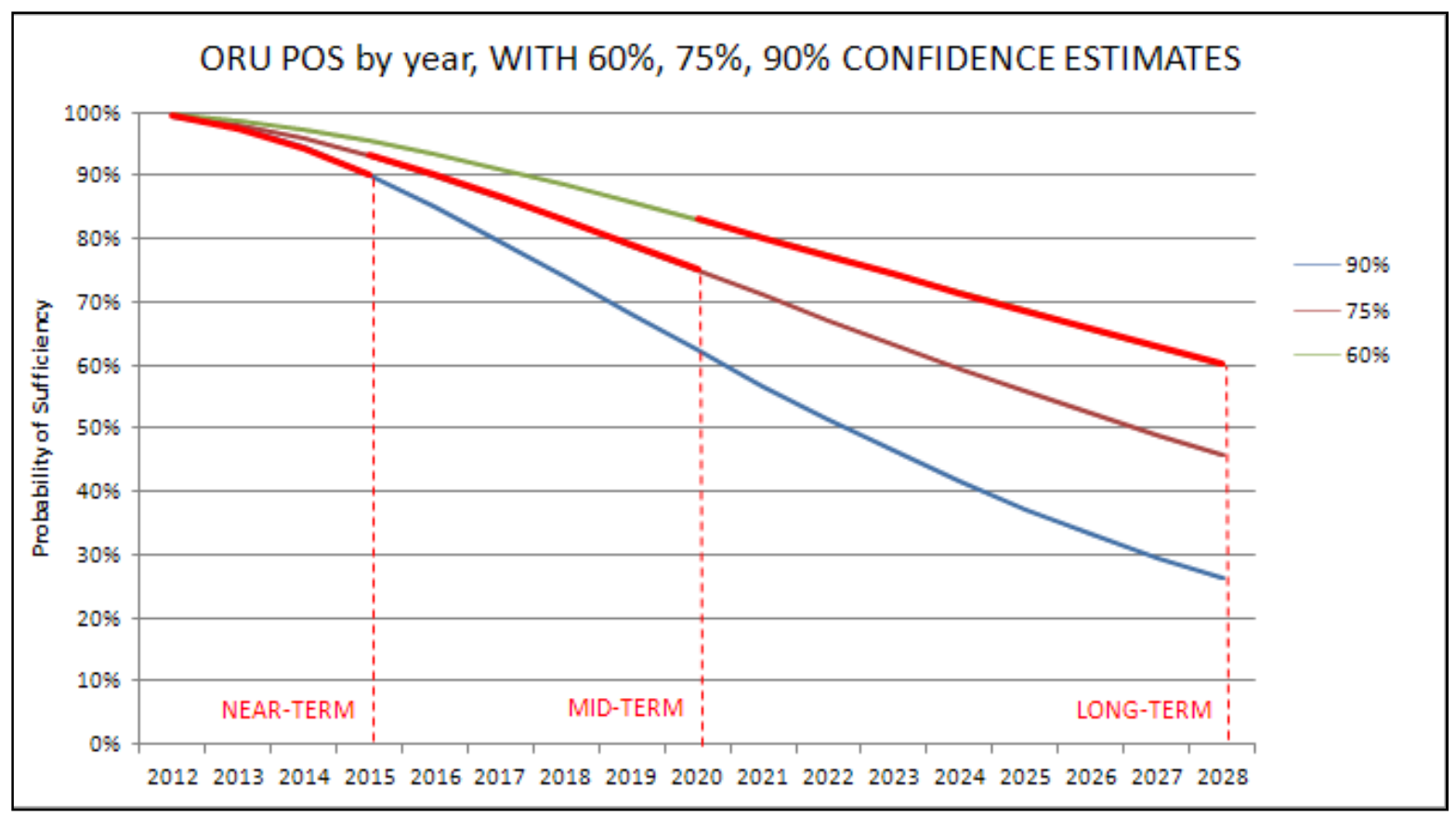

Figure 8. Generic ORU time-series with varying near, mid, and long-term probability and confidence targets. Figure shows what number of 'events' sparing quantities should cover to meet the targets: 90/90 TARGET: 1.36 events / 1 additional spare, 75/75 TARGET: 1.02 events/ 1 additional spare, 60/60 TARGET: 0.91 events / no additional spares 
We expect the values for ORU POS and confidence not to focus only on time. We should expect discussion to consider for instance ORU criticality, redundancy, the ability to recover the system in event of ORU failure, and other contingencies. A dynamic approach to setting POS and confidence levels provides a consistent result per ORU in terms of a sparing assessment. In the above example, $L \& M$ will provide a sparing projection in the near-term of needing a total of 2 spares, compared to a mid-term projection of 2 spares, and a long-term projection of 1 spare. When introduced to PACT evaluation, L\&M has also considered an alternative approach to setting targets in the probability/confidence trade-space. It may be more appropriate to hold the ORU POS constant over time and instead relax only our confidence in the estimate. The reasoning is that longer time horizons exhibit greater epistemic uncertainty in the true ORU failure rate. Consider the sensitivity, Figure 9, case where we hold 90\% POS fixed throughout the near, mid, and long-term projection.

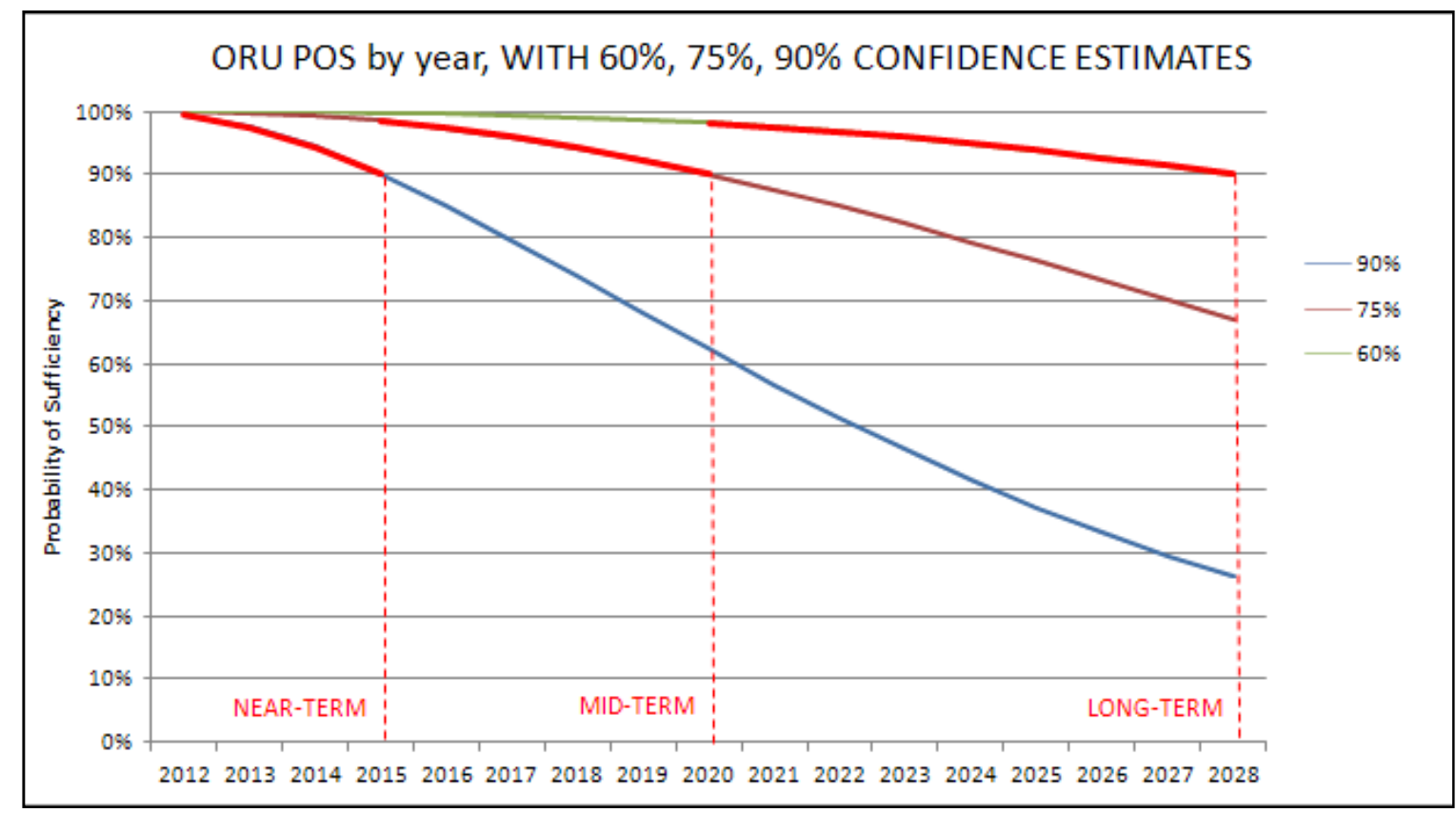

Figure 9. Generic ORU time-series with varying near, mid, and long-term confidence targets and fixed probability. Figure shows what number of 'events' sparing quantities should cover to meet the targets: 90/90 TARGET: 1.36 events / 1 additional spare, 90/75 TARGET: 1.79 events / 1 additional spare, 90/60 TARGET: 2.31 events / 2 additional spares

We fully expect the exact targets for ORU POS and confidence to change according to function, and possibly on an ORU basis. We have noted the dramatic impact in accounting for epistemic uncertainty when establishing probability and confidence levels given projection in time. How does the PACT Evaluation represent our risk posture should our level of certainty in the ORU failure rate increase? The following data illustrates this aspect.

An alternative sensitivity case demonstrates the same sparing assessment for the hypothetical ORU; only decreased epistemic uncertainty is represented by lowering the EF from 4 to 1.5. In the near-term, only 0.87 events are needed to be covered by a spare. The analysis indicates the existing spare is sufficient. In the mid-term, 1.67 events are projected, indicating demand for one additional spare. In the long-term, the number of events increases to 2.69 , requiring 2 additional spares. The decreased uncertainty does not necessarily result in a consistent trend over time. Using PACT Evaluation, we can adjust our sparing recommendation according to our level of uncertainty in the ORU failure rate. 


\section{Conclusion}

When introducing PACT Evaluation, we define and account for the epistemic uncertainty characterizing ISS sparing assessments to more accurately represent the true risk of sparing for the ISS. We acknowledge what we can realistically know about an ORU's true failure rate given the various methods used to predict ORU reliability. We describe how the lack of complete knowledge directly relates to our perceived risk in vehicle sustaining plans. We have reviewed the current approach developed by L\&M for conducting sparing assessments. The approach takes the latest Bayesian update in ORU failure rate, and applies a POS value given a number of spares. When applying high confidence levels, this approach results in inflation of the number of spares to meet a target when the target does not change over time. Furthermore, we cannot explicitly calculate our epistemic uncertainty. Instead, we introduce that by adopting a Lognormal process we are able to integrate the ORU Posterior EF from the Bayesian process to assess a level of confidence in the ORU POS estimate. This defines the trade-space inherent to the PACT Evaluation approach and thus we measure our epistemic uncertainty. We can also control the POS and confidence levels for sparing assessments. We would like to add mention of our investigation into the meaning of a combined unit of Probability and Confidence where a single measure of the area under the curve (see 'Area of Sufficiency' Figure 5, Section IV) may indicate epistemological probability within the tradespace. This line of work is on-going.

Our near-term work is to recommend new targets to the ISS community. This work includes the following key areas:

- $\quad$ For a given component or group of components or spares, trading a target probability of sufficient spares with the confidence in that target probability.

- Once that trade is complete defining the recommended POS and confidence for a component and/or group of components.

- $\quad$ Setting unique POS and confidence targets as a function of time horizon. For example if the time to turn on a supplier is long for a critical component you would be less willing to relax the POS than for a less critical component or spare that can be easily manufactured or even substituted.

- Making recommendations on the number of spares required once the target POS and confidence has been set and representing and communicating the risk to the stakeholders.

Our research to date has not addressed assessing ORUs modeled in a functional hierarchy. In such cases we understand epistemic uncertainty propagates throughout the modeled systems. We have tools to do so and look forward to further developing this evaluation method to achieve our goal: to model every ISS ORU accounting for epistemic uncertainty to provide the program the best representation of sparing demand and better sustain the vehicle through 2020 and beyond.

PACT is a useful methodology and has been proposed to supplement ISS sparing analysis. As with the current process, PACT Evaluation should be input to extensive coordination with expert and program stakeholders for decision-making. Preliminary results indicate a valuable trade space for selecting probability of sufficiency and confidence targets. 


\section{Appendix A - Six ORU Case Study Results Charts}

\section{ORU Selection Criteria for Case Study}

\begin{tabular}{|c|c|c|}
\hline Selected ORU & ORU Function & Rationale for Selection \\
\hline $\begin{array}{l}\text { Remote Power } \\
\text { Control } \\
\text { Mechanism } \\
\text { (RPCM T5 Ext) }\end{array}$ & $\begin{array}{l}\text { The RPCM receives commands and transmits data via an } \\
\text { internally redundant MIL-STD-1553B Data Bus. Type } 5 \\
\text { will function as a remote high power switching ORU } \\
\text { module consisting of two } 12 \text { AMP switching and sixteen } \\
3.5 \text { AMP switches. Each switch will provide short circuit } \\
\text { and overload shutdown. }\end{array}$ & $\begin{array}{l}\text { Large population of } \\
\text { installed units and spares } \\
\text { providing rich operational } \\
\text { experience including } \\
\text { failures and redesign }\end{array}$ \\
\hline $\begin{array}{l}\text { Improved } \\
\text { Payload } \\
\text { Ethernet Hub } \\
\text { Gateway } \\
\text { (IPEHG) }\end{array}$ & $\begin{array}{l}\text { IPEHG is an ORU replacement for the existing Payload } \\
\text { Ethernet Hub/Gateway (PEHG). }\end{array}$ & $\begin{array}{l}\text { Small internal ORU, no } \\
\text { operational experience }\end{array}$ \\
\hline $\begin{array}{l}\text { Pump Module } \\
\text { Assembly } \\
\text { (PMA) }\end{array}$ & $\begin{array}{l}\text { The primary function of the Pump Module is to circulate } \\
\text { liquid ammonia around the thermal loops (ETCS) for } \\
\text { acquisition of heat loads. }\end{array}$ & $\begin{array}{l}\text { Large external ORU, } \\
\text { critical hardware with } \\
\text { many hours of operation } \\
\text { and a random failure }\end{array}$ \\
\hline $\begin{array}{l}\text { Hydrogen } \\
\text { Dome }\end{array}$ & $\begin{array}{l}\text { It is contained in a dome, operates near vacuum, and is } \\
\text { designed to contain fire or explosion in cell stack. The } \\
\text { hydrogen dome also provides second barrier to hydrogen } \\
\text { leakage. }\end{array}$ & $\begin{array}{l}\text { Large internal ORU, no } \\
\text { redundancy, relatively little } \\
\text { operational experience }\end{array}$ \\
\hline $\begin{array}{l}\text { Solar Array } \\
\text { Rotary Joint } \\
\text { Drive Lock } \\
\text { Assembly Drive } \\
\text { Lock Assembly } \\
\text { (SARJ-DLA) }\end{array}$ & $\begin{array}{l}\text { The DLA continuously positions the panels as directed by } \\
\text { the Rotary Joint Motor Controller (RJMC). The DLA } \\
\text { consists of the drive motor assembly (DMA), and the } \\
\text { engage/disengage mechanism (EDM). }\end{array}$ & $\begin{array}{l}\text { External ORUs with } \\
\text { redundancy, similar } \\
\text { function differing failure } \\
\text { rates, SARJ DLA modeled } \\
\text { in the Usable Power } \\
\text { function hierarchy }\end{array}$ \\
\hline $\begin{array}{l}\text { Thermal } \\
\text { Radiator Rotary } \\
\text { Joint Drive } \\
\text { Lock Assembly } \\
\text { Drive Lock } \\
\text { Assembly } \\
\text { (TRRJ-DLA) }\end{array}$ & $\begin{array}{l}\text { The TRRJ DLA continuously positions the radiator panels } \\
\text { as directed by the Rotary Joint Motor Controller (RJMC). } \\
\text { The DLA consists of the drive motor assembly (DMA), and } \\
\text { the engage/disengage mechanism (EDM). }\end{array}$ & $\begin{array}{l}\text { External ORUs with } \\
\text { redundancy, similar } \\
\text { function differing failure } \\
\text { rates, TRRJ DLA modeled } \\
\text { as a separate ORU }\end{array}$ \\
\hline
\end{tabular}



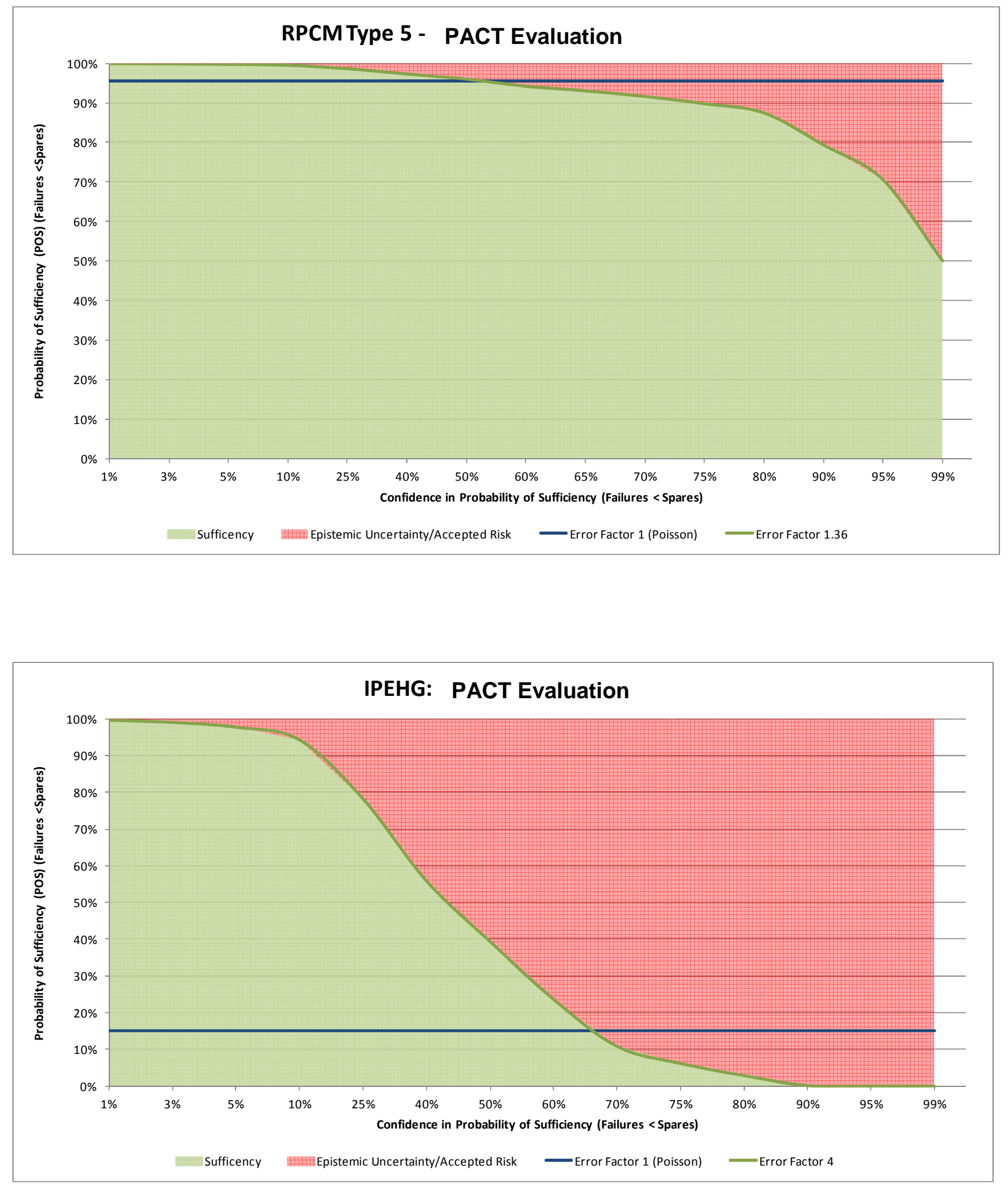

American Institute of Aeronautics and Astronautics 

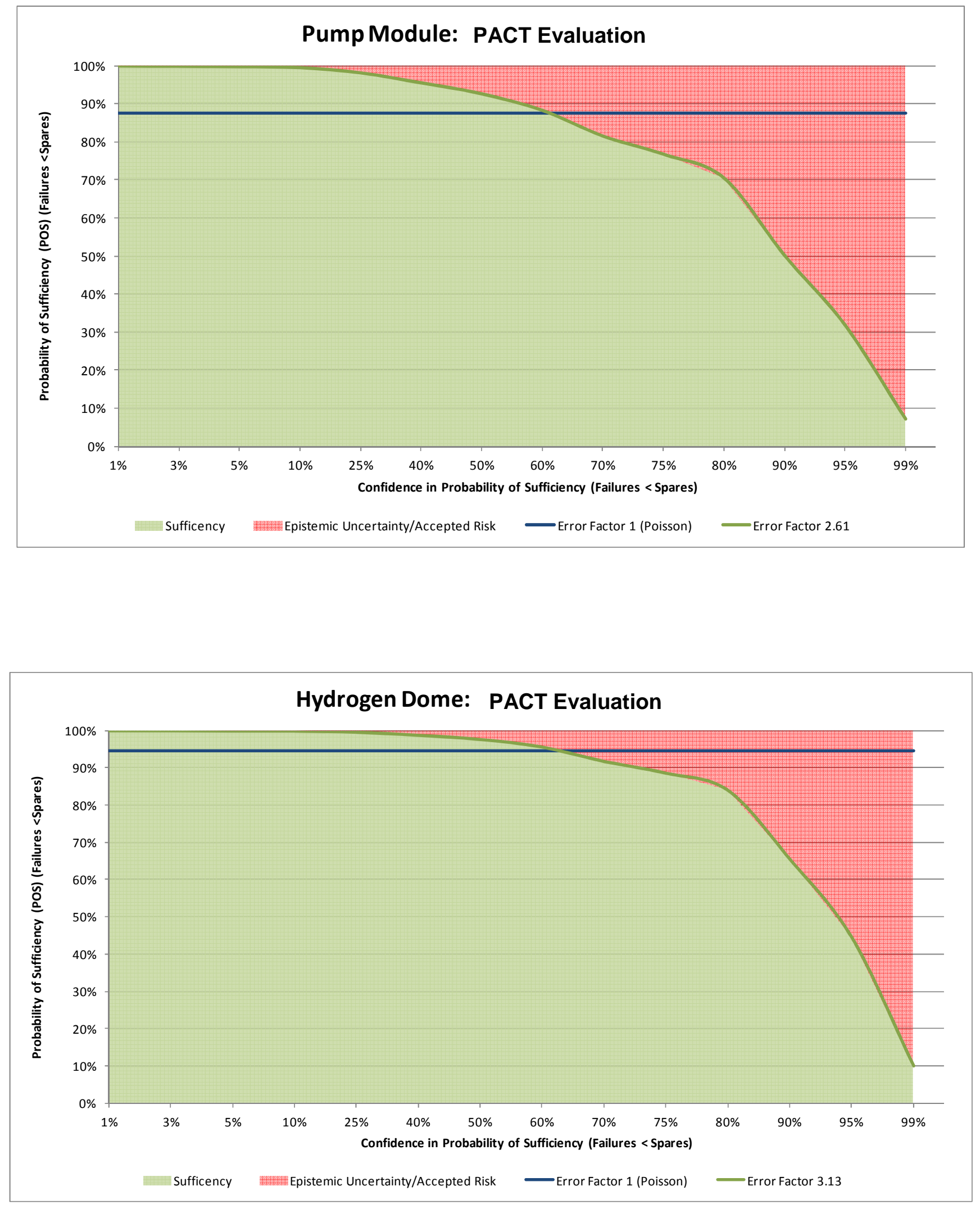

American Institute of Aeronautics and Astronautics 

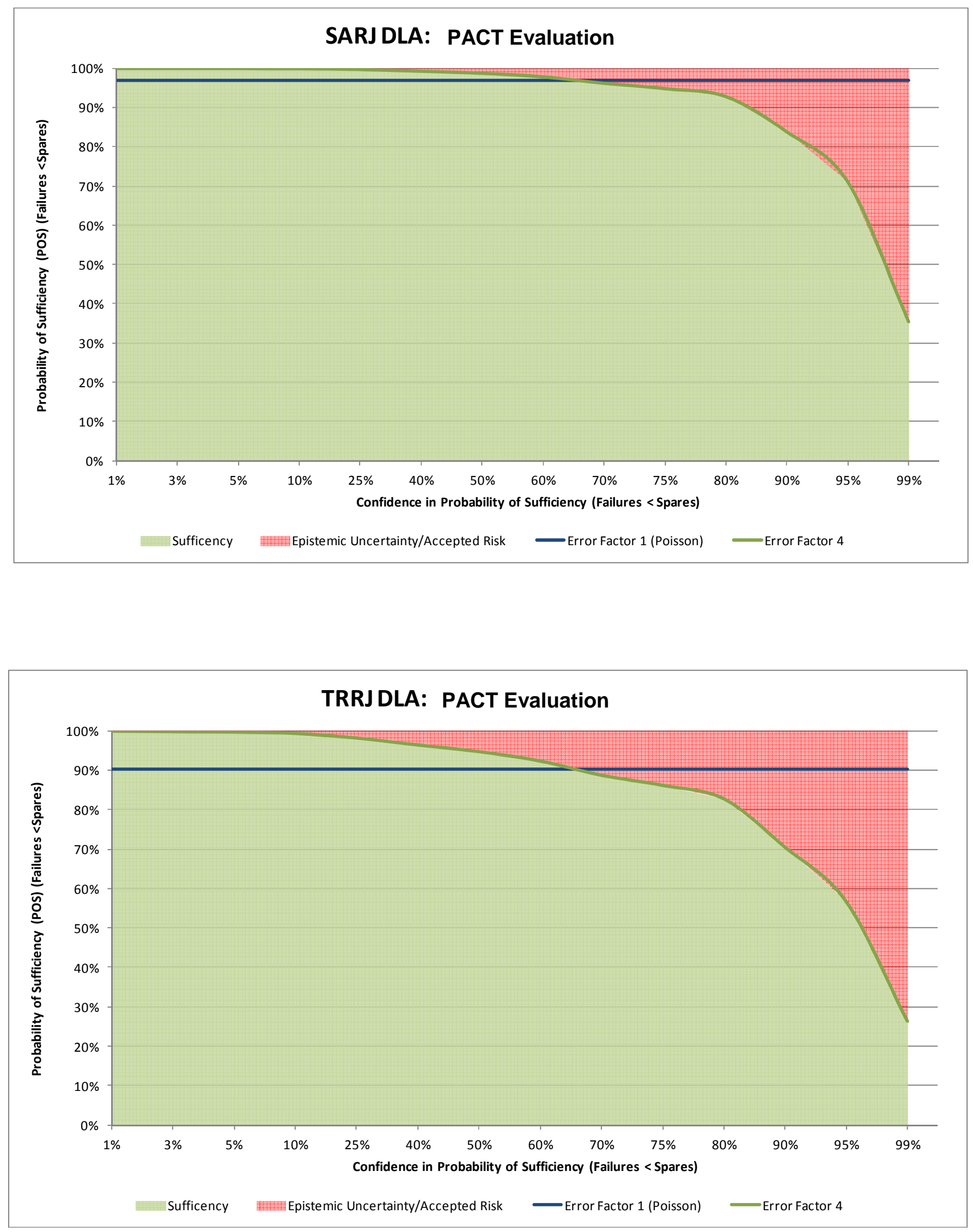

American Institute of Aeronautics and Astronautics 


\section{Appendix B - Acronyms}

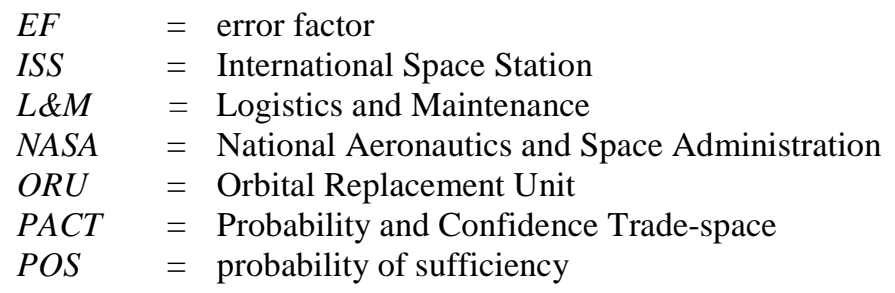

\section{Acknowledgments}

The authors of this paper extend our utmost gratitude to Dr. William E. Vessely, of NASA Headquarters, whose technical expertise, advice, and encouragement was a catalyst to our efforts for completing this phase of our work.

\section{Works Cited}

1. Stamatelatos, M. and Dezfuli, H. Probabilistic Risk Assessment Procedures Guide for NASA Managers and Practitioners. Washington DC : NASA, 2011. NASA/SP-2011-3412,.

2. NASA Spare Parts Acquisition w/Change 2 (12/19/08). 2008. NASA Procedural Requirements, NPR 5900.

3. Department of Defense. Military Handbook: Reliability Prediction of Electronic Equipment. 1990.

4. International Space Station: Ongoing Assessments for Life Extension Appear to be Supported. [Online] April 2011. [Cited: July 6, 2012.] http://www.gao.gov/new.items/d11519r.pdf.

5. Technical Probabilistic Risk Assessment (PRA) Procedures for Safety and Mission Success for NASA Programs and Projects. 2010. NASA Procedural Requirement, NPR 8705.5A-TOC .

6. Imagery Online via DIMS. [Online] NASA, May 29, 2011. [Cited: June 21, 2012.] Photo taken by STS-134 crew member on space shuttle Endeavourafter the ISS and shuttle began their post-undocking relative separation.. https://issimagery.jsc.nasa.gov/io/app/info.cfm?pid=21760714. s134e011548. 